\title{
Book Review: Guhl, Pascal (2018): Die Entwicklung neuer Stadtquartiere aus städtebaulicher Sicht. Analyse der Projekte seit 1990
}

\section{Essen: Klartext-Verlag. = Dortmunder Beiträge zur Raumplanung 148. 366 Seiten}

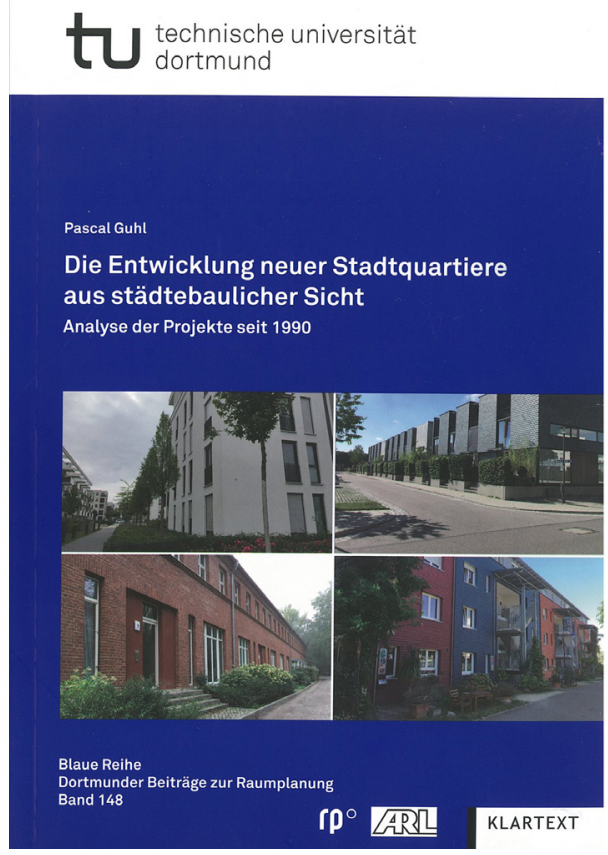

Der Titel des Buches macht neugierig. Es handelt sich um eine ambitionierte Dissertation im Studiengang Raumplanung der Technischen Universität Dortmund. Die Arbeit besteht aus zwei Teilen, von denen jeder einzelne, entsprechend aufbereitet, für eine Promo- tion gereicht hätte. Den Kern bildet eine Vollerhebung der neuen Stadtquartiere, die seit 1990 in Deutschland geplant und gebaut wurden. Darauf aufbauend werden vier gezielt ausgewählte größere Stadtquartiere vorgestellt und vergleichend ausgewertet: Frankfurt-Riedberg, München-Messestadt Riem, Potsdam-Bornstedter Feld und Freiburg-Rieselfeld. Von besonderem fachlichen Interesse ist der erste Teil, während sich der zweite Teil mit Fallstudien in eine beachtliche Zahl ähnlicher Quartiersanalysen - meist ebenfalls Dissertationsschriften einfügt, worauf der Verfasser auch verweist und die er punktuell aufgreift (S. 17).

Die Vollerhebung und Analyse der seit der Wiedervereinigung entstandenen Stadterweiterungs- und Stadtumbauquartiere ist eine äußerst verdienstvolle Unternehmung. Hierzu gab es bisher nur die veralteten und auch hinsichtlich des Erhebungsaufwandes eher bescheidenen und deshalb lückenhaften Studien des Bundesamtes für Bauwesen und Raumordnung von 2007, aktualisiert im Jahre 2012. Dabei wäre es jetzt ein Vierteljahrhundert nach Beginn der zweiten großen Stadterweiterungswelle mehr als angezeigt, analog zum Großsiedlungsbericht von 1994 aus der Perspektive der Städtebaupolitik und der Stadtforschung Rechenschaft über die in dieser Zeit meist nach dem neuen Leitbild der kompakten, nutzungsgemischten Stadt entstandenen

\footnotetext{
*Corresponding author: Prof. Dr. Johann Jessen, Relenbergstraße 46, 70174 Stuttgart, Deutschland,
}

E-mail: johann.jessen@si.uni-stuttgart.de 
Quartiere abzulegen. In diese Lücke stößt die Arbeit von Pascal Guhl. Sie kann sie aber, das sei vorweg gesagt, nicht schließen - dies wäre von einer Dissertation wohl auch zu viel erwartet.

Als Stadtquartiere sind hier städtebauliche Projekte definiert, die mindestens vier Hektar groß sind, zwei Baufelder umfassen, Wohnnutzung einschließen, eine öffentliche Infrastruktur aufweisen und einer einheitlichen Planungskonzeption folgen (S. 40/41). Damit sind sie nach unten gegen komplexe Einzelimmobilien abgegrenzt, nach oben gibt es dagegen keine Abgrenzung. Diese sehr geräumige Quartiersdefinition reicht potenziell von einem 4 ha großen Gebiet mit knapp über 100 freistehenden Einfamilienhäusern und einem Kinderspielplatz bis zum flächengrößten Quartier der Fallstudien München-Messestadt Riem mit 560 ha. Abgesehen davon, dass der Verfasser keine Begründung für die 4-ha-Untergrenze liefert, zahlt er für diese weite Definition, die an eine Flächenvorgabe geknüpft ist, einen hohen Preis. Hätte er die Definition und die Auswertung näher an die des Großsiedlungsberichtes angelehnt, wäre es ihm leichter möglich gewesen, seine Ergebnisse in einen historischen Kontext zu stellen, und er hätte in zentralen Dimensionen die Großsiedlungen der 1950er und 1960er Jahre mit den Quartieren der 1990er-Jahre vergleichen können. Außerdem erschwert die äußerst hohe innere Heterogenität der Grundgesamtheit die Analyse und Interpretation der Daten, vor allem, wenn diese, wie hier der Fall, sehr lückenhaft sind.

Der Titel des Buches untertreibt, was selten ist. Die Quartiere werden keineswegs nur in städtebaulicher Hinsicht, sondern vor allem in organisatorischer, planungskonzeptioneller und prozessualer Hinsicht beschrieben und analysiert. Die besonderen städtebaulichen Qualitäten werden dagegen, selbst in den Fallstudien, eher nur gestreift. Da kommt der Verfasser in der Darstellung der Gemeinsamkeiten und Unterschiede ohne zusätzliche Pläne, Zeichnungen und Fotos aus. Ein Lageplan muss den Leserinnen und Lesern reichen.

Den jeweiligen empirischen Darstellungen stellt er den Stand der Forschung zu Stadtquartieren sowie zu den Instrumenten, Verfahren und Prozessen der Quartiersentwicklung voran. Wie häufig in Dissertationen zeigen diese teilweise weit ausholenden Passagen, wie tief der Verfasser in die Materie eingedrungen ist. Aber leider auch für diese Dissertationsschrift gilt, dass Empirie und Theorie unverbunden nebeneinander stehen, also weder die Theorie die empirischen Fragen anleitet, noch die empirischen Befunde dazu beitragen, die Theorie zu bereichern.
Durch Internet- und Literaturrecherche ermittelte der Verfasser 435 Quartiere, die seiner Quartiersdefinition entsprechen. In seine Analyse bezieht er die 271 Quartiere ein, die nach 1990 begonnen wurden und bis 2015 beendet waren. Für sie erstellt er aus den verfügbaren Unterlagen einen Datensatz von rund 70 Variablen, die er durch gezielte Nacherhebungen bei den Gemeindeverwaltungen ergänzt hat. Der mit enormem Arbeitsaufwand erstellte Datensatz ist gleichwohl äußerst lückenhaft, nur bei 13 der 70 Merkmale liegen für mehr als 200 Quartiere die Informationen vor, für fast die Hälfte der Merkmale liegen die Besetzungszahlen unter 100 (S. 26-28). Mit anderen Worten: Der Datensatz ist nur sehr begrenzt belastbar. Hinzu kommt, dass es offensichtlich auch keine Plausibilitätskontrollen der Datensätze gegeben hat. Anders ist nicht zu erklären, dass in Tabellen (S. 99/100) das Quartier Neckarsulm-Amorbach II mit 7.300 Wohneinheiten nach München-Riem als das nach Zahl der Wohnungen größte nach 1990 fertiggestellte Quartier in Deutschland ausgewiesen wird. Wenn das zuträfe, hätte sich das zuvor in der Fachwelt herumgesprochen. Hier wurde vermutlich eine Eins als Sieben gelesen, ohne dass es jemand bis zur Drucklegung gemerkt hat. So etwas erhöht nicht das Vertrauen in die Befunde.

Gleichwohl kommt die umfassende statistische Auswertung zu einer Fülle plausibler Ergebnisse, auf die dort, wo sie sich auf hohe Besetzungszahlen stützt, auch Verlass sein dürfte. Um nur einige zu nennen: Der Schwerpunkt der Entwicklung neuer Quartiere lag in den Wachstumsregionen, dort vor allem in den Großstadtregionen; die neuen Quartiere in den wachstumsschwächeren Regionen entstanden „aus städtebaulichen Gründen" vornehmlich auf Militär- und Industriebrachen. Zwei Drittel der Quartiere fanden sich in den integrierten Lagen, vor allem in der Innenstadt und am Innenstadtrand. Es zeigt sich, dass das Leitbild "Innenentwicklung vor Außenentwicklung" durchaus praktische Wirkung entfaltet hat. Je größer die Stadt, desto größer sind nach Fläche und Zahl der Wohnungen im Mittel die Quartiere, desto höher die Baudichte und ihr Anteil in integrierten Lagen. Je größer das Quartier, desto länger die Projektlaufzeit absolut, desto kürzer der relative Zeitaufwand für die Entwicklung pro Wohn- oder Flächeneinheit. Auch hier greift die economy of scale. Unabhängig von der Größe werden Stadtquartiere „auf der grünen Wiese“ schneller fertig und sind kostengünstiger als Stadtumbauvorhaben. Die städtebauliche Entwicklungsmaßnahme ist das am häufigsten angewendete Instrument und weist auch im Mittel die kürzesten Projektlaufzeiten im Verhältnis zur Projektfläche auf. Entgegen der Erwar- 
tung wurde eine Nutzungsmischung in den Quartieren nur in kaum mehr als der Hälfte umgesetzt, auch hier vornehmlich in den innerstädtischen Lagen, während sie in den Stadterweiterungen fast keine Rolle spielt. Allerdings geben hier die Daten keine nähere Auskunft zu der besonderen Körnigkeit der Nutzungsmischung und der Diskrepanz zwischen der ursprünglich geplanten und der tatsächlich erreichten Nutzungsmischung, wie überhaupt dieser eigentlich naheliegende Aspekt des Grades der Zielerreichung bzw. die Differenz zwischen Plan und Realisierung keine größere Rolle in der Untersuchung spielt. Insgesamt leiden zahlreiche inhaltliche Aussagen zum Städtebau und zur Planung der Quartiere unter den niedrigen Besetzungszahlen, die die Zuverlässigkeit der Ergebnisse grundsätzlich in Frage stellen. So kommt die Auswertung zu dem erstaunlichen oder besser erschütternden Ergebnis, dass 45 Prozent der Projekte nicht in übergeordnete gesamtstädtische Konzepte eingebunden waren (S. 209). Zum einen liegen hier nur die Zahlen für 94 Projekte vor, zum anderen bleibt völlig unklar, was genau damit gemeint ist bzw. ab wann ein Projekt als in übergeordnete Konzepte eingebunden gelten kann. Auf die Auskunft der Gemeinden dürfte sich diese Aussage nicht stützen können, denn es ist kaum vorstellbar, dass Gemeinden dies bei 45 Prozent der Projekte dieser Größenordnung einräumen würden. Der Autor selbst gibt keine Hinweise darauf, wie er den Begriff der Einbindung operationalisiert hat und wie er sich diesen krassen Befund erklärt.

Nicht nur der Datensatz ist begrenzt belastbar, auch bleibt dessen Auswertung offensichtlich unter dem, was er eigentlich hergäbe. Der Verfasser führt eine Fülle von Korrelationsanalysen durch, die vor allem von der Verfügbarkeit auswertbarer Daten geleitet sind und deren Ergebnisse sich nur schwer deuten lassen. Die Darstellung der statistischen Ergebnisse wirkt oft wenig professionell. Es werden Histogramme erstellt, wo einfache Tabellen zum Ziel geführt hätten. Auch gibt es leicht tilgbare Schnitzer. So werden Mittelwerte, zum Beispiel für die Wohnungszahl in den Quartieren, in den Tabellen grundsätzlich mit vier Stellen hinter dem Komma angegeben. Gern hätte man in einem Anhang die wichtigsten Erhebungsinstrumente gesehen.

Es bleibt ein sehr zwiespältiger Eindruck zurück. Einerseits verdienen das hohe Engagement, das enorme Arbeitspensum und die hochinteressante Fragestellung den ganzen Respekt, auf den ambitionierte wissenschaftliche Qualifizierungsarbeiten einen Anspruch haben, zumal wenn sie sich wie hier einem dringenden Desiderat zuwenden. Andererseits muss die Frage gestellt werden, ob die Herausgeberschaft einer ange- sehenen und traditionsreichen Publikationsreihe wie der Dortmunder „Blauen Reihe“ nicht darauf hätte dringen müssen, dass die Arbeit für die Buchpublikation noch einmal sehr gründlich inhaltlich wie redaktionell bearbeitet und gestrafft wird. 